\title{
True technology-enabled mental health care: trust, agency and ageing
}

\author{
Sarah Burch $^{1}$, Claire Preston ${ }^{1}$, Sarah Bateup ${ }^{2}$ \\ ${ }^{1}$ Anglia Ruskin University, Cambridge, UK; ${ }^{2}$ Ieso Digital Health, Cambridge, UK \\ Correspondence to: Dr. Sarah Burch. Anglia Ruskin University, Faculty of Health, Social Care and Education, Young Street Site, East Road, \\ Cambridge, CB1 1PT, UK. Email: sarah.burch@anglia.ac.uk. \\ Provenance: This is an invited article commissioned by Editor-in-Chief Dr. Steven Tucker, MD (Tucker Medical, Singapore). \\ Response to: Williams A. True technology-enabled mental health care: when will we trust the computers and robots? Mhealth 2018;4:10.
}

Received: 21 June 2018; Accepted: 02 August 2018; Published: 07 August 2018.

doi: $10.21037 /$ mhealth.2018.08.02

View this article at: http://dx.doi.org/10.21037/mhealth.2018.08.02

Technology and ageing is a subject which attracts intense interest. In part, this is undoubtedly due to the search for a solution which will help manage the scale, costs and predicted impact of demographic change (1). However, it arguably also reflects a fascination with imagining futures, both in personal and social terms. This may be why there tends to be a focus on the more exotic technological applications. The current emphasis on Ambient and Assisted Living (AAL) contains many pragmatic developments, but can also be used to conjure up exciting visions of older people inhabiting a futuristic domestic landscape staffed by robots. In practice, it is important that this prospect is not allowed to overwrite the more prosaic yet hugely beneficial gains which technology can bring to people who may require increasing levels of support or who struggle to access current-day services.

As noted by Williams (2), to date mental health has not attracted the same enthusiasm for technological innovation as other areas of life. This may stem from the way in which older people's mental health problems are characterised as inevitable, intractable or invisible, in spite of numerous studies showing that there can be good responses to treatment across a range of conditions $(3,4)$. Amongst the array of computer-aided mental health interventions available, internet-enabled cognitive behaviour therapy (IECBT) stands out in that it is not primarily designed to save resources or limit therapist input. It delivers therapistled sessions in real time which are facilitated through an online portal utilizing written communication (see www.iesohealth.com). Kessler et al. (5) have previously demonstrated its effectiveness as a mode of treatment, whilst our analysis of secondary data suggests it has considerable potential as an intervention for older people (6).

There are several ways in which IECBT may be able to make an important contribution to the care and support available to older people. One message from our secondary data analysis was that older people who self-referred for IECBT were assessed as experiencing a wider range of conditions than those who were referred by medical professionals such as GPs (6). This raises some interesting considerations. In terms of how referrals operate, individual clinical commissioning groups (CCGs) in England decide whether to commission IECBT as a service. Patients who reside in areas where IECBT is commissioned may either self-refer or be referred by a medical professional. Once referral or self-referral takes place, the person is assessed by a therapist and the primary diagnosis recorded. The fact that people referred by medical professionals have a narrower range of resultant diagnoses than those who selfrefer warrants further exploration. However, a plausible interpretation is that medical professionals either miss certain mental health problems or regard them as unlikely to respond to treatment, hence they only refer a narrow group of older people. Given that studies have shown that older people can be both under-diagnosed and undertreated in relation to conditions like depression (7), this suggests that facilitating self-referral may be one way in which to counter this tendency within services.

Increasing the agency and autonomy of older people may be facilitated in other ways. Allowing self-referral for treatment does not have to be confined to IECBT as opposed to more traditional modes of talking therapies. Yet 
one distinctive component of IECBT can be particularly valuable to older people, namely that it provides a permanent written record of the therapy session which has been conducted through text-based communication. This gives the older person the ability to review sessions, which not only reinforces an intrinsic element of the cognitive behavioural therapeutic approach, but also facilitates ownership of the process along with supporting recall of the discussion. Thus technology reinforces the therapeutic exchange.

It is often assumed that communication facilitated by technology will be somehow inferior to all face to face contact. This fear is particularly acute when it comes to talking therapies, as there is such a premium placed on the centrality of the therapeutic relationship. However, it is a misapprehension that relationships can only thrive in person $(8,9)$. Whilst it may not be welcomed by everyone, others can find both anonymity and solace in a relationship conducted at one remove. The computer screen echoes the screen of the confessional, providing a layer of privacy which affords freedom to speak of difficult emotions. Mental health problems are still perceived as sufficiently stigmatizing for this to be a positive boon of technology.

Privacy at a distance can also be accompanied by accessibility (9). One of the biggest difficulties for older people can be physically getting to services even once referred. IECBT means that there is no need to arrange transport or negotiate unfamiliar locations or buildings, as therapy can be accessed at home via the computer. Ageing in place is a common aspiration for older people, yet places can be outgrown or become increasingly unsuitable for their occupants. For ageing in place to be meaningful, the place itself may need to be transformed.

Computer-facilitated therapy has, as previously noted, usually been seen as a cheap option. Most forms are variants on guided self-help, which has the appeal of being far less resource-intensive than conventional therapy. This is not the case with IECBT, as it involves as much therapist input as face to face methods. It is a fallacy that technology will always deliver cost-savings, just as it turned out to be a fallacy that community care would always be cheaper than institutional care, at least not if it were to be delivered to a high standard. Nevertheless, there is still the potential for a reduction in the use of resources, however the resources conserved are those of the older people themselves rather than the NHS. Someone who can access therapy from home does not have to arrange transport, ask family members for help getting to the treatment setting or use up their valuable energy on getting themselves out of the house for another medical appointment rather than a chosen activity. Ageing can involve a dwindling of many kinds of personal and financial resources. If technology enables people to receive therapy at home, these resources can be preserved and even boosted by better mental health. IECBT provides the opportunity to enhance the quality of care provided so that more people benefit from improved mental health. Computer assisted clinical decision support can be used with IECBT to provide information and guidance to therapists whilst they are providing a therapy session. For example, IECBT has had promising results in the lab using interventions that support therapists to provide the right diagnosis and treatment with the aim of enabling more patients to get better.

Of course, IECBT will not suit or work for everyone, whatever age they are. Our research (6) indicates that only a minority of older people access IECBT. Many will not be technologically adept, will find typing slow or difficult, or will value the social contact of face to face interaction. However, if Improving Access to Psychological Therapies (IAPT) as an initiative is to succeed in its goal of increasing uptake amongst older people (10), making effective use of technology is an important strategy to embrace. Broadening the ability to self-refer will permit the increasing numbers of older people who feel comfortable with communicating online to access services which can be transformative in their ability to relieve mental distress. This is an exciting area of study which merits further research.

\section{Acknowledgements}

None.

\section{Footnote}

Conflicts of Interest: The authors have no conflicts of interest to declare.

\section{References}

1. Schulz R, Wahl HW, Matthews JT, et al. Advancing the Aging and Technology Agenda in Gerontology. Gerontologist 2015;55:724-34.

2. Williams A. True technology-enabled mental health care: when will we trust the computers and robots? Mhealth 2018;4:10.

3. Laidlaw K, Kishita N. Age-appropriate augmented 
cognitive behaviour therapy to enhance treatment outcomes for late-life depression and anxiety disorders. GeroPsych 2015;28:57-66.

4. Cuijpers P, van Straten A, Smit F, et al. Is psychotherapy for depression equally effective in younger and older adults? A meta-regression analysis. Int Psychogeriatr 2009;21:16-24.

5. Kessler D, Lewis G, Kaur S, et al. Therapist-delivered Internet psychotherapy for depression in primary care: a randomised controlled trial. Lancet 2009;374:628-34.

6. Burch S, Preston C, Bateup S et al. The use of internetenabled cognitive behavioural therapy in the treatment of depression and anxiety amongst older people. Int J Aging

doi: 10.21037/mhealth.2018.08.02

Cite this article as: Burch S, Preston C, Bateup S. True technology-enabled mental health care: trust, agency and ageing. mHealth 2018;4:30.
Soc 2017;8:1-11.

7. Overend K, Bosanquet K, Bailey D, et al. Revealing hidden depression in older people: a qualitative study within a randomised controlled trial. BMC Fam Pract 2015;16:142.

8. Suler J. The online disinhibition effect. Cyberpsychol Behav 2004;7:321-6.

9. Preston C, Moore S. Ringing the changes: the role of telephone communication in a helpline and befriending service targeting loneliness in older people. Ageing Soc 2018; doi:10.1017/S0144686X18000120

10. Prina AM, Marioni RE, Hammond GC, et al. Improving access to psychological therapies and older people: findings from the Eastern Region. Behav Res Ther 2014;56:75-81. 